\title{
In Situ Synthesis of Carbon Nanotubes on Heated Scanning Probes Using Dip Pen Techniques
}

\author{
RoHit V. GARgATE and DEBJyoti BANERJEe \\ Department of Mechanical Engineering, Texas A\&M University, College Station, Texas, USA
}

Summary: Carbon nanotubes (CNT) were synthesized on heated scanning probes and under ambient conditions without requiring Chemical vapor deposition (CVD) apparatus or process gases. In this study, dip pen nanolithography (DPN) techniques were utilized for deposition of catalyst precursors on the scanning probe tips in the form of aqueous solution of metal salts - prior to the synthesis of the CNT. A layer of fullerene $\left(\mathrm{C}_{60}\right)$ of $\sim 200 \mathrm{~nm}$ thickness was vapor

Address for reprints: Debjyoti Banerjee, Department of Mechanical Engineering, Texas A\&M University, College Station, TX 77843-3123, USA e-mail: dbanerjee@tamu.edu

Received 9 October 2007; Accepted with revision

11 December 2007

Contract/grant sponsor: Micro Technology Office (MTO) of the Defense Advanced Research Projects Agency (DARPA).

Contract/grant sponsor: Space and Naval Warfare Center (SPAWAR).

Contract/grant sponsor: Office of Naval Research (ONR).

Contract/grant sponsor: Mechanical Engineering Department at Texas A\&M University and the Texas Engineering Experimentation Station (TEES).

Contract/grant sponsor: National Science Foundation; Contract/grant number: DBI-0116835.

Contract/grant sponsor: National Science Foundation; Contract/grant number: CBET Grant No. 0630703.

Contract/grant sponsor: Micro/Nano-Fluidics Fundamental Focus Center (DARPA-MF3).

Contract/grant sponsor: New Investigations Program (NIP 2005) of the Texas Space Grants Consortium (TSGC).

Contract/grant sponsor: Office of Naval Research (ONR).

Contract/grant sponsor: Air Force Office of Scientific Research (AFOSR).

Contract/grant sponsor: American Society for Engineering Education (ASEE).

Contract/grant sponsor: Summer Faculty Fellowship (SFFP)

Program.

Contract/grant sponsor: Air Force Research Labs (AFRL).

Contract/grant sponsor: Army Research Office (ARO) SBIR

Phase II; Contract/grant number: Lyntech Inc.

Contract/grant sponsor: National Science Foundation (NSF)

SBIR Phase I.

Contract/grant sponsor: Nano-MEMS Research LLC. deposited on the scanning probe tip prior to the deposition of the metal catalyst. During the in situ synthesis of the CNT on the scanning probes, the temperature of the heated scanning probes reached $350-400{ }^{\circ} \mathrm{C}$. Hence the scanning probes were heated in an inert atmosphere to prevent potential oxidation of the deposited fullerene layer. The synthesized CNTs were subsequently characterized using SEM and Raman spectroscopy. The Raman spectroscopy showed peaks in the Radial breathing mode (RBM), as well as the defect (D) and graphitic (G) bands. The RBM peaks indicate that the single walled carbon nanotube (SWCNT) ranged in diameter from $0.9-1.5 \mathrm{~nm}$. The peaks in the Raman spectra are indicative of SWCNT mixtures (metallic and semconducting) and possibly multiwalled carbon nanotube (MWCNT). Hence this process can be optimized to synthesize SWCNT of a specific chirality (metallic or semiconducting). This study differs from an earlier study reported in the literature involving synthesis of CNT on scanning probes where the process temperatures typically exceeded $700{ }^{\circ} \mathrm{C}$, and resulted in synthesis of highly graphitic MWCNT (Sunden, et al., 2006). SCANNING 30: 151-158, 2008. (C) 2008 Wiley Periodicals, Inc.

Key words: carbon nanotubes, dip pen nanolithography, MEMS, nanotechnology, Raman spectroscopy

PACS: $73.63 . F g$ Nanotubes

\section{Introduction}

Carbon nanotubes (CNT) are known to form naturally during the combustion processes (Singer and Grumer, 1959; Su and Chen, 2007) - though their initial discovery was attributed to the arc discharge process on graphite electrodes (Iijima, 1991). This technique was refined (Hafner et al., 1999) and subsequently several other processes were developed for manufacturing CNT in "bulk" quantities - such as "CVD" (Ebbesen and Ajayan, 1992), and the HiPCo 
process (Ouellette, 2002). However, these processes yield a mixture of small proportions of MWCNT and SWCNT while the major proportions of the reaction products are carbon fibers and soot. Also, in these processes the synthesized SWCNT is obtained as a mixture of metallic and semiconducting type. Hence, conventional techniques require expensive separation processes, which are an impediment to their implementation in novel commercial devices that have been envisioned for decades (Robertson, 2007).

Applications of CNTs have been envisioned in superconductors, field-effect transistors (FETs), memory elements, MEMS (microelectromechanical systems), micro/nanosensors and micro/nanoelectronic devices (Robertson, 2007). Microelectronic applications require precise location and chirality of synthesized CNTs with high spatial density. Hence successful implementation of SWCNT in nanoelectronic circuits has not been possible using current synthesis techniques primarily due to the following three factors (Robertson, 2007): (i) the inability to pattern SWCNT at a precise location, (ii) the inability to precisely control the chirality of the synthesized SWCNT, i.e. metallic or semiconducting type (separation of SWCNT mixtures is expensive and complicated), and (iii) the inability to synthesize SWCNT with high spatial number density.

In earlier attempts (Sunden et al., 2006) nanotube bundles were synthesized on heated microcantilevers using CVD techniques. An iron catalyst layer of 10-nm thickness was vapor deposited on the microcantilever followed by self heating of the cantilever to $800^{\circ} \mathrm{C}$ in a quartz tube that is typically used for CVD, and in the presence of organic precursors of CNT (e.g. methane and acetylene). Graphitic nanotube bundles were synthesized on the microcantilever, which was reported to be verified by Raman spectroscopy.

In this paper, we introduce a method for the synthesis of CNT, wherein no CVD is required, and the precise positioning of the synthesized CNT is managed through the strategic placement of metal catalysts on a MEMS platform. The MEMS platform consists of a microcantilever scanning probe tip integrated with a microheater at the base of the microcantilever. Thus this technique obviates some significant hurdles presented by other methods (Robertson, 2007) that prevent the exploitation of CNT in micro/ nanoelectronics. In this study, we report the synthesis of CNT on commercially available heated scanning probe tips (or "probes"). The catalyst precursors were deposited in a "wet" form on the probes from the aqueous solution of metal salts using dip pen techniques. The metal salts served as precursors of the catalysts required for synthesis of CNT. To obviate the CVD process, fullerene precursors were vapor deposited on the probes. The probes were selfheated using the microheaters located at the base of the scanning probes to enable the synthesis of CNT from fullerenes. Thus, this process did not require any process gases for the synthesis of CNT.

\section{Materials and Methods}

\section{Experimental Apparatus and Procedure}

A scanning probe platform consisting of a microcantilever array with integrated microheaters at the base was used for in situ synthesis of CNT. The heated microcantilevers used in this study consists of an array of eight microcantilevers (ActivePen system, Manufactured by Nanoink Inc.). The silicon nitride microcantilevers are 100 microns long, with 20 microns width and on a pitch of 40 microns. Each cantilever has an electronic serpentine gold heating element at the base, a gold heat spreader (10 microns by 60 microns), which facilitates the heat distribution, and a pyramid shaped oxide sharpened probe at the tip. Figure 1 shows the magnified image (100X) of a microcantilever from the array. The scanning probe tip is located at the distal end and the microheater is fabricated at the base of the microcantilever.

The Mbraun Labmaster double glove box system with gas purification (located at the Center for Nanomanufacturing, University of Texas, Austin); was

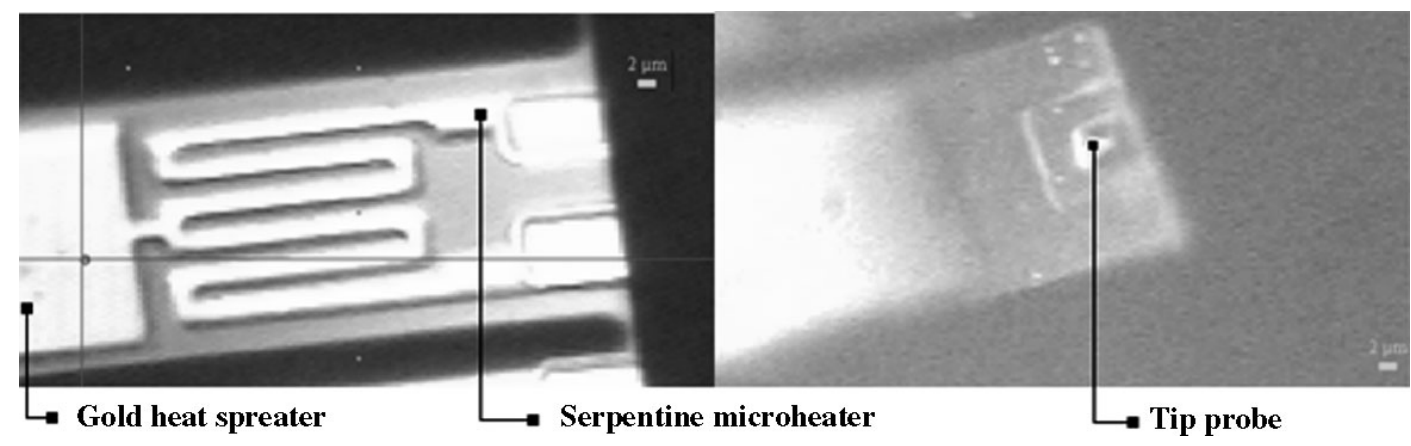

Fig 1. The serpentine heating element (Left) on a microcantilever of the scanning probe platform. Magnified image of the scanning probe tip (Right). 
R. V. Gargate and D. Banerjee: In situ synthesis of carbon nanotubes using dip pen techniques

used for the vapor deposition of fullerene (C-60) onto the active pens. This instrument consists of a twochamber glove box system. A spin coater (specialty coating systems model P6700) is available inside the smaller glove box chamber. An Edwards thermal deposition chamber with four sources is available inside the larger glove box. A $150{ }^{\circ} \mathrm{C}$ vacuum oven is mounted at one end of the glove box. The ActivePen is placed in the thermal deposition chamber and the $\mathrm{C}_{60}$ fullerene powder (Manufactured by Nano-C Inc., Westwood, MA) was placed in the target container. The target container was electrically heated at a pressure of $1 \mathrm{mTorr}$ to a temperature of $550{ }^{\circ} \mathrm{C}$ to enable sublimation of the fullerene powder. The deposition rate by sublimation was estimated to be $\sim 20 \mathrm{~nm} / \mathrm{s}$ for a total deposition time of $10 \mathrm{~s}$. The fullerene thickness was estimated to be $\sim 200 \mathrm{~nm}$ at the end of the vapor deposition process using a quartz microbalance.

A passive microfluidic platform (Inkwells) was used to deposit metal catalysts onto the scanning probe microcantilever array using an atomic force microscope (AFM) platform. Various experimental parameters such as the position of the active probes, actuation current, deposition speeds, deposition patterns, etc., can be varied and controlled through the interfacing software. An Inkwell is a microfluidic platform for delivering solutions of specific chemical precursors to an array of scanning probe tips for their subsequent deposition using dip pen techniques (Banerjee et al., 2005; Rosner et al., 2006). In the Inkwell device, an array of reservoirs connect to microwells through microchannels. A hydrophobic layer is deposited on the top of the substrate to prevent any cross-contamination.
After mounting the scanning probe array on the AFM, the individual microcantilevers can be addressed and actuated by passing current through the respective microheaters at the base. Using this scheme each tip can be retracted or extended and all the tips can be actuated individually or simultaneously. At the beginning of the synthesis experiments all the microcantilevers in the array are actuated sequentially to verify if any of the probes are damaged. The deflection of each cantilever is observed through the real time video window of the interfacing software.

The apparatus is placed in an environmental control chamber, which is connected to the nitrogen supply line from a pressurized nitrogen cylinder. The instrument is equipped with temperature and humidity sensors and an exhaust fan for controlling the humidity of the environment surrounding the probes mounted on the instrument. The values of temperature and humidity can be varied, controlled and monitored through the control software.

After vapor deposition of the fullerene layer, Xray photoelectron spectroscopy (XPS) was used to characterize the array of scanning probe tips on the ActivePen. Figure 2 shows the representative XPS data for a scanning probe tip. A distinct peak at $286 \mathrm{eV}$ is observed on all the tips confirming that the tips are coated with a layer of fullerene (Cox et al., 1991; Chiang et al., 1992; Mochida et al., 1995).

\section{Dipping Procedure}

Several metals, which form carbide eutectic are known to catalyze CNT growth, such as, Pd, Pt,

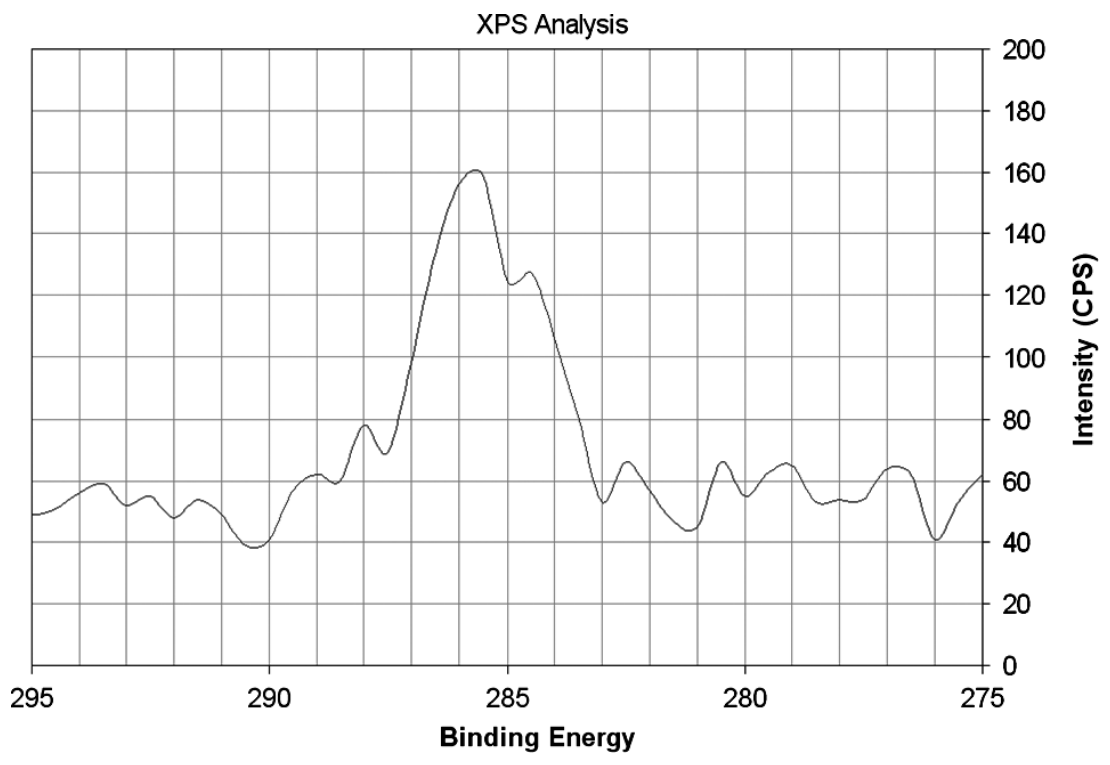

Fig 2. An XPS analysis of the scanning probes tips showing a peak at $286 \mathrm{eV}$, which confirms the presence of the sublimated coating of fullerene. 
$\mathrm{Ni}, \mathrm{Co}, \mathrm{Fe}$, etc. (Li et al., 2001). In this study, the individual probes were coated with aqueous solutions of three different metal salts as catalyst precursors (for $\mathrm{Pd}, \mathrm{Co}$ and $\mathrm{Ni}$ ). These catalysts precursors were chosen due to their low reduction temperatures to form bare metal from the salt. The catalyst precursors were obtained from $50 \mathrm{mM}$ aqueous solutions of $\mathrm{NiCl}_{2}$, $\mathrm{CoCl}_{2}$ and $\mathrm{PdCl}_{2}$. These salt solutions were used to dip coat the fullerene-coated scanning probes. Inkwells are necessary for the purpose of dipping the probe tips in the desired solution and for confining the dip coating over a localized area on the scanning probe tips. The Inkwell used in this study consists of six reservoirs connected to six microwells through separate microchannels. The catalyst precursor in the form of an aqueous solution of the metal salt is used for coating the scanning probe tips. The salt solutions were obtained by mixing a metal salt with water in a 10$\mathrm{mL}$ Eppendorf tube, followed by decanting the salt solution using a micropipette. A droplet of the salt solution $(50-100 \mathrm{~nL})$ is deposited in the reservoir using a syringe. The salt solution flows from the reservoir into the microwell by microcapillary wicking in the microchannels. The Inkwell is then mounted in the AFM. The scanning probes were then mounted on the AFM, and vertically aligned on top of a filled microwell in the Inkwell.

Individual probes are sequentially dipped into respective microwells for coating each tip with a specific catalyst precursor. The aligned cantilever was then lowered into the microwell in steps of 3-5 microns. The dipping of the scanning probe tip was detected when the cantilever snaps into the microwell due to capillary forces. The scanning probe is pulled into the microwell instantly when it makes contact with the liquid meniscus. This can also be observed in the video window of the interfacing software by a change in the reflectivity of the cantilever compared to the other microcantilevers in the array. Figure 3 shows a schematic of the scanning probe tip dipped into the microwell. Figure 4 shows the cantilever array before and during the dipping process.

The dipping procedure was repeated sequentially for all the eight microcantilevers. Of the eight microcantilevers in the ActivePen array, three of the scanning probe tips were dipped into $\mathrm{NiCl}_{2}$ solution, three were dipped in $\mathrm{CoCl}_{2}$ solution and two were dipped in $\mathrm{PdCl}_{2}$ solution. Performing the dipping operation using the microwells helps to constrain the area over which the catalyst is deposited in each scanning probe tip.

After accomplishing the dipping of the scanning probe tips into the catalyst precursor solutions, the Inkwell was dismounted. The humidity was recorded to be $42 \%$ indicating ambient moisture and air was in contact with the probe tips. Hence, Nitrogen was passed into the chamber, and the relative humidity was monitored. When the humidity dropped to $\sim 1 \% \mathrm{RH}$ after approximately $15 \mathrm{~min}$, indicating that the environmental chamber was filled with nitrogen, the synthesis experiments were performed sequentially for each cantilever. Initially, the current supply to an individual microheater (at the base of a microcantilever) was set at $25 \mathrm{~mA}$. The current was increased in the steps of 2-3 $\mathrm{mA}$, until the limiting value of $50 \mathrm{~mA}$ was attained for the supply current (the software setting limited the supply current to this value). This resulted in a heat spreader of the microcantilever attaining a temperature of $\sim 400^{\circ} \mathrm{C}$, while the scanning probe tip reached a temperature of $\sim 350^{\circ} \mathrm{C}$ (temperature data was obtained using infrared microscopy measurements). The supply current to the microheater at the base of each microcantilever was maintained at $50 \mathrm{~mA}$ for a period of 5 min and the power was then switched off. This procedure was repeated sequentially for all the scanning probe tips, before dismounting the probe array.

The low synthesis temperature provides several advantages. It has been reported that lower synthesis temperatures yield a narrower distribution of the diameters (and correspondingly the chirality) of the

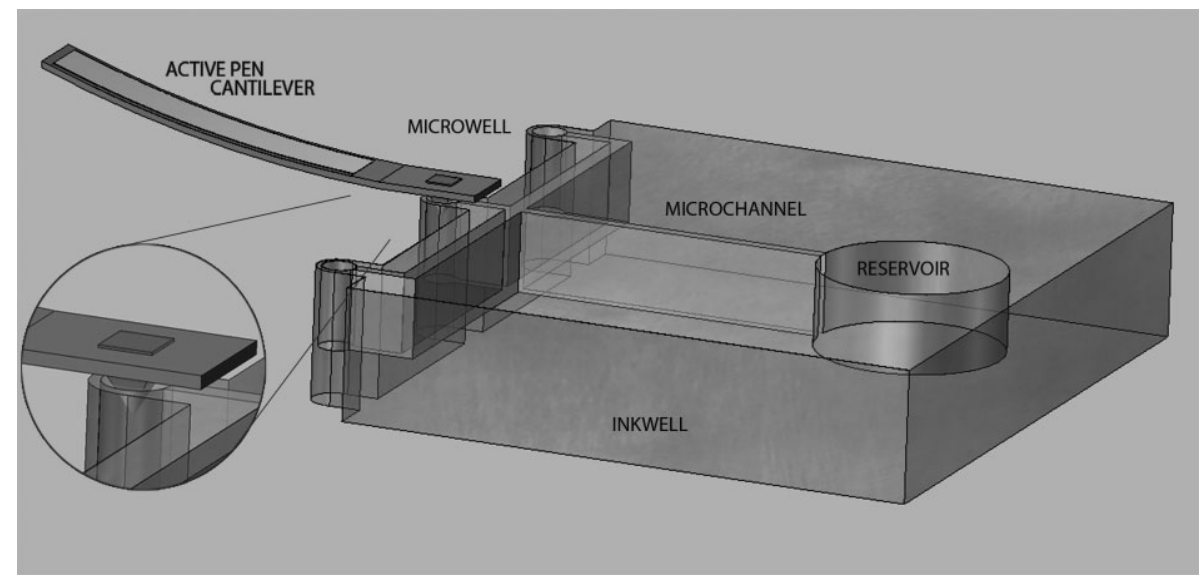

Fig 3. A schematic showing a microcantilever with the scanning probe array dipped into a microwell of the Inkwell. 

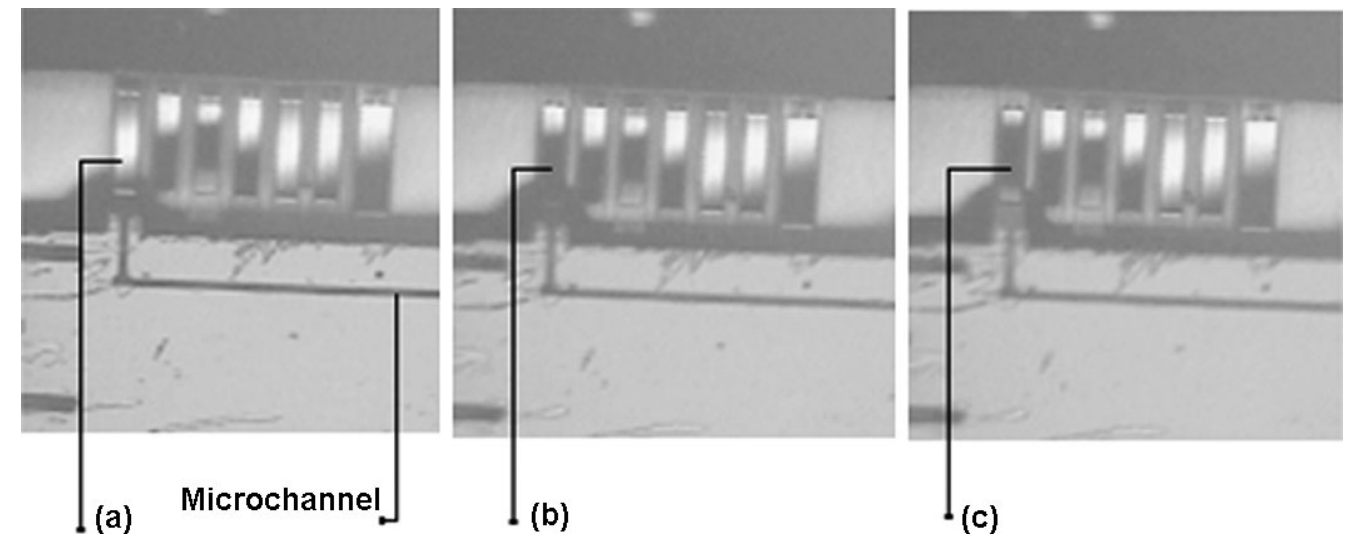

Fig 4. Inkwell dipping procedure. (a) A microcantilever in the scanning probe array is vertically aligned on top of a microwell, before dipping. The microwell contains the salt solution as the catalyst precursor. (b) The cantilever probe lowered halfway toward the microwell. (c) The cantilever dipped into the microwell. The change of reflectivity compared to adjacent microcantilevers shows the deflection of the microcantilever and indicates successful dipping of the scanning probe tip into the liquid meniscus.

synthesized CNT (Robertson, 2007). Also, lower synthesis temperatures can enable easier integration with conventional microelectronics manufacturing and packaging processes.

\section{Results}

The materials characterization was performed to verify the synthesis of CNT achieved from heating fullerene in the presence of the metal catalysts. It is expected that the catalyst precursors (e.g. $\mathrm{PdCl}_{2}$ ) will reduce to form pure metal (e.g. Pd) when heated in presence of fullerenes. The metal catalyst will then nucleate CNT from the fullerenes. Scanning electron microscopy (SEM) was performed to verify if successful synthesis of materials was achieved on the scanning probe tips. Figure 5 shows the SEM image of the scanning probe tip after coating with fullerenes. Figures 6 and 7 shows the SEM image of the scanning probe tip after the synthesis experiments were completed with $\mathrm{PdCl}_{2}$. At lower resolution the SEM image (Figure 6) shows that precipitated particles were observed on the scanning probe. At a higher resolution (Figure 7), thread-like structures are observed at the base of the scanning probe tip-potentially indicating the formation of CNT bundles. The diameter of the bundles is observed to be between $50-100 \mathrm{~nm}$. The SEM images for the other scanning probe tips were similar to Figure 6 . The maximum operating (accelerating) energy of the SEM was $40 \mathrm{kV}$ and the images were obtained from secondary electron emission. However, at higher resolution other structures were observed in Figure 7, which were not detected on the other scanning probe tips. These additional nanostructures (as shown in Figure 7) consist of "hair-like"or "wire" type of structures. These nanostructures were subsequently verified to be SWCNT bundles by micro-Raman spectroscopy.

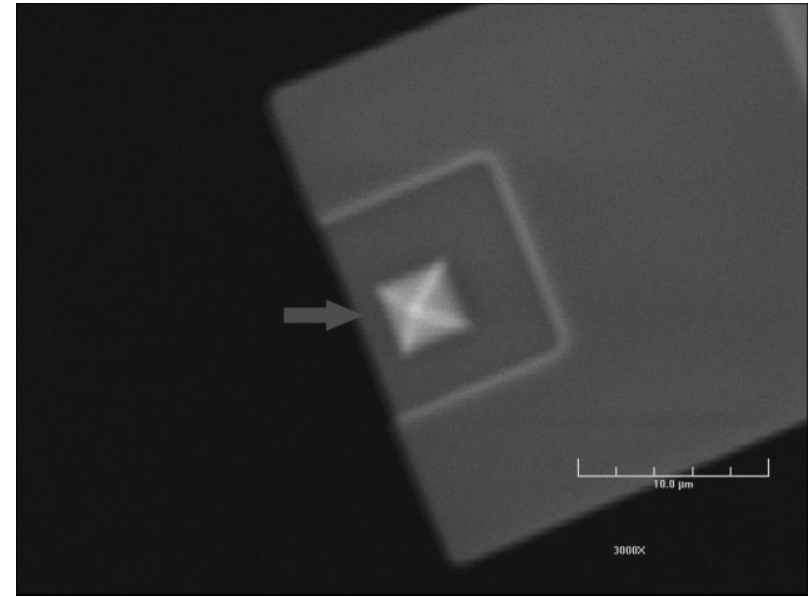

Fig 5. An SEM image of a microcantilever before the dipping and synthesis experiments. The pyramid shaped oxide sharpened scanning probe tip is shown in this figure.

Figure 7 shows that the spatial density of the synthesized CNT is sparse. Such sparse density is useful for studying the field-emission characteristics from individual CNT as well as for measuring the thermophysical characteristics of individual CNT. This synthesis technique provides the feasibility study for such novel characterization studies. Energy dispersive X-ray spectroscopy ("EDS") was performed along with SEM imaging of the tips following the synthesis experiments. Table I lists the results from the EDS study. The results show that the tip surface is coated with an abundance of carbon (potentially as fullerene or carbon nanotubes) and small quantities of palladium (which is a remnant of the $\mathrm{PdCl}_{2}$ solution deposited by dip coating).

Subsequently, micro-Raman Spectroscopy was performed to verify the quality of the synthesized CNT 


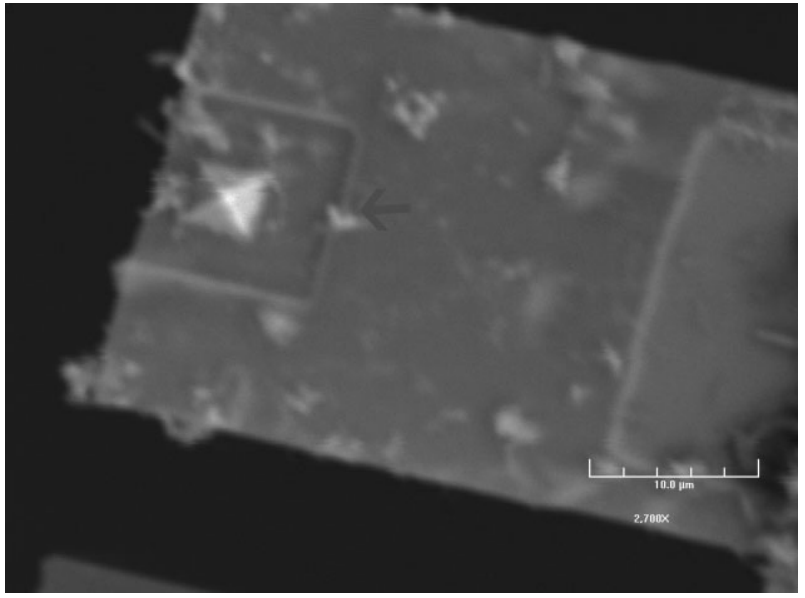

Fig 6. An SEM image of a microcantilever after the synthesis experiments. The SEM images are obtained at different magnifications. The figure shows catalyst particles formed on the microcantilever after the dipping operation.

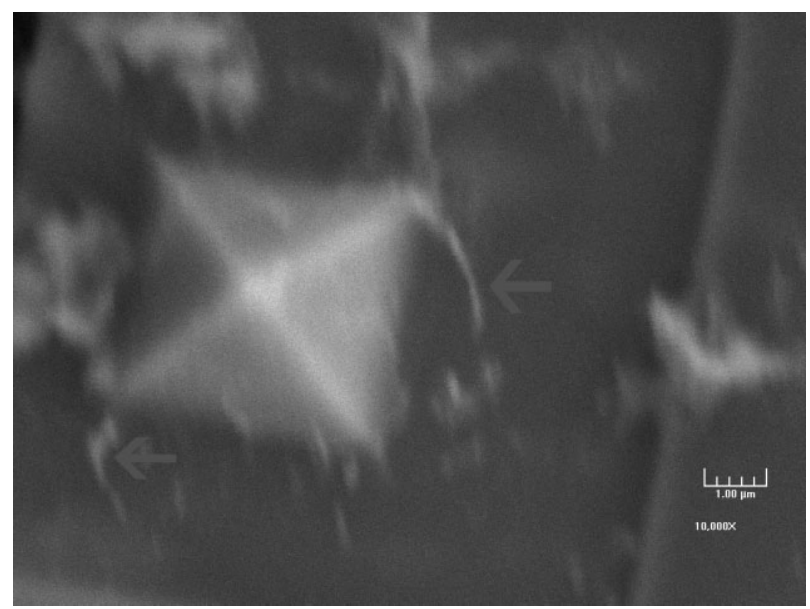

Fig 7. An SEM image of a microcantilever after the synthesis experiments. The figure shows carbon nanotube bundles, which appear as hair or wire shaped material synthesized at the base of the scanning probe.

bundles (Model: LabRam IR Confocal Raman Spectroscopy-Microscopy Instrument, Manufacturer: JY Horiba, Japan). The instrument is equipped with a 635$\mathrm{nm}$ wavelength laser with a probe area of 1 micron $^{2}$. This laser was used for the optical excitation of the material synthesized on the probes, and each cantilever was analyzed sequentially. In the Raman spectra, the quality of the synthesized CNT was ascertained by tracing the peaks in the radial breathing mode (RBM), the defect (D) and the graphitic (G) bands. The scanning probe array was mounted on a glass slide for performing Raman spectroscopy.

The RBM mode in Raman spectra is unique to cylindrical symmetry, and is widely used to determine
TABLE I. Energy dispersive X-ray spectroscopy (EDS) analyses of the microcantilever tip after carbon nanotube synthesis

\begin{tabular}{lc}
\hline Element & Weight $(\%)$ \\
\hline $\mathrm{Si}$ & 0.00 \\
$\mathrm{C}$ & 96.85 \\
$\mathrm{Pd}$ & 3.15 \\
$\mathrm{Ni}$ & 0.00 \\
$\mathrm{Co}$ & 0.00 \\
Total & 100.00 \\
\hline
\end{tabular}

the diameter of SWCNT. Distinct peaks for RBM, $\mathrm{D}$ and $\mathrm{G}$ were obtained for the two tips coated with $\mathrm{PdCl}_{2}$ while distinct peaks were not observed for the other scanning probe tips. This indicates that synthesis of CNT using $\mathrm{PdCl}_{2}$ is feasible at lower synthesis temperatures (i.e. $350-400{ }^{\circ} \mathrm{C}$ ). The Raman spectra of the scanning probe tips coated with $\mathrm{PdCl}_{2}$ are shown in Figures 8 and 9.

Figure 8 shows that in the micro-Raman spectra RBM peaks are observed in the range $150-300 \mathrm{~cm}^{-1}$ for the scanning probe coated with $\mathrm{PdCl}_{2}$ catalyst precursor and fullerene. Since the microcantilevers are composed of silicon nitride, a peak is also observed at $300 \mathrm{~cm}^{-1}$ corresponding to silicon. In the RBM mode distinct peaks were seen at 167, 180, 232, 253, and $275 \mathrm{~cm}^{-1}$.

The diameter $(d)$ of the SWCNT can be derived from the location of the Raman shift peaks $(\omega)$ in the RBM mode by the following equation (Jorio et al., 2001):

$$
\mathrm{d}=\frac{248}{\omega}
$$

where the units of $d$ is in $\mathrm{nm}$ and $\omega$ is in $\mathrm{cm}^{-1}$. For the range of peaks observed in our experiments - this corresponds to SWCNT of diameters ranging from 0.9 to $1.5 \mathrm{~nm}$.

Typically, Raman spectra reported in the literature that the D band is observed at a Raman shift of $\sim 1350 \mathrm{~cm}^{-1}$ and the $\mathrm{G}$ band at $\sim 1600 \mathrm{~cm}^{-1}$. If the $\mathrm{D}$ band is more prominent (higher or comparable intensity) than the $\mathrm{G}$ band, it is indicative of presence of MWCNT. Conversely, if the G band is significantly more prominent with respect to the $\mathrm{D}$ band, along with the presence of RBM bands it is indicative of SWCNT (Jorio et al., 2001; Jorio et al., 2004; Thomsen et al., 2004).

Figure 9 shows that for the $\mathrm{PdCl}_{2}$ there is a peak corresponding to the $\mathrm{D}$ band at $1350 \mathrm{~cm}^{-1}$ and a peak corresponding to the $\mathrm{G}$ band at $1582 \mathrm{~cm}^{-1}$. This confirms that $\mathrm{PdCl}_{2}$-coated scanning probes catalyze CNT growth at lower temperatures. Lower processing temperatures were also reported in the literature to provide a narrower range of chirality distribution for SWCNT 


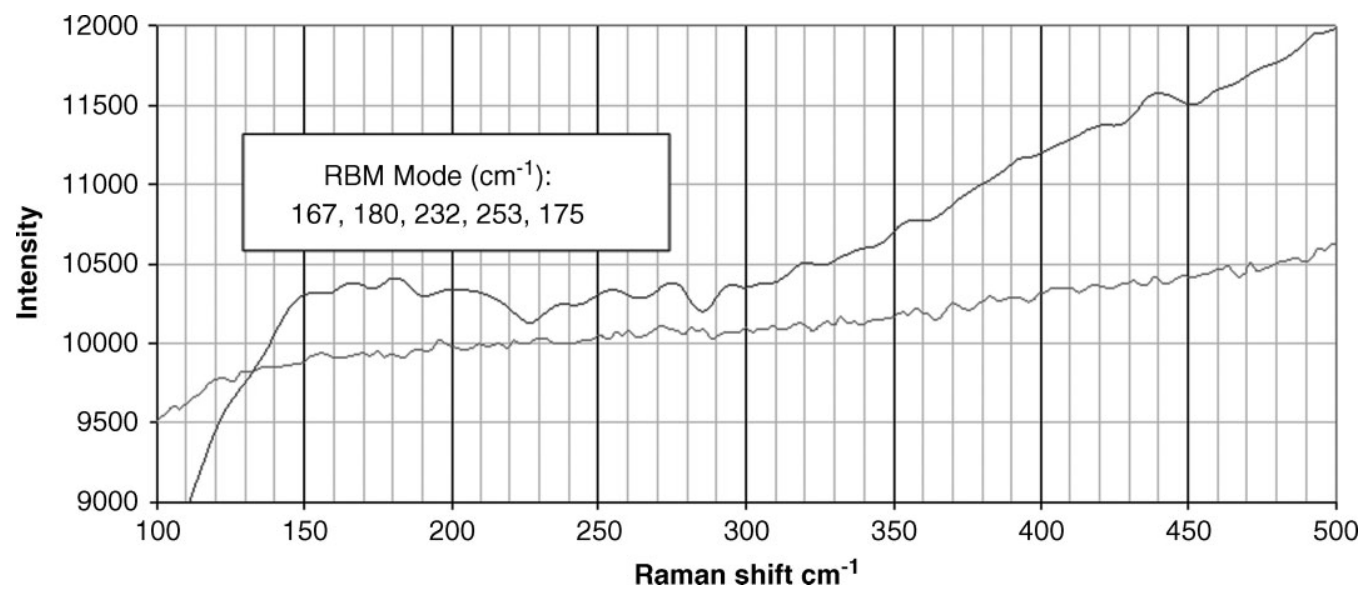

Fig 8. Micro-Raman spectra in the RBM mode of the fullerene-coated scanning probe tip (dipped in $\mathrm{PdCl}_{2}$ ), before (red) and after (blue) the synthesis experiment. The graphs show peaks at the following locations in the RBM mode $\left(\mathrm{cm}^{-1}\right): 167,180,232,253$, and 275.

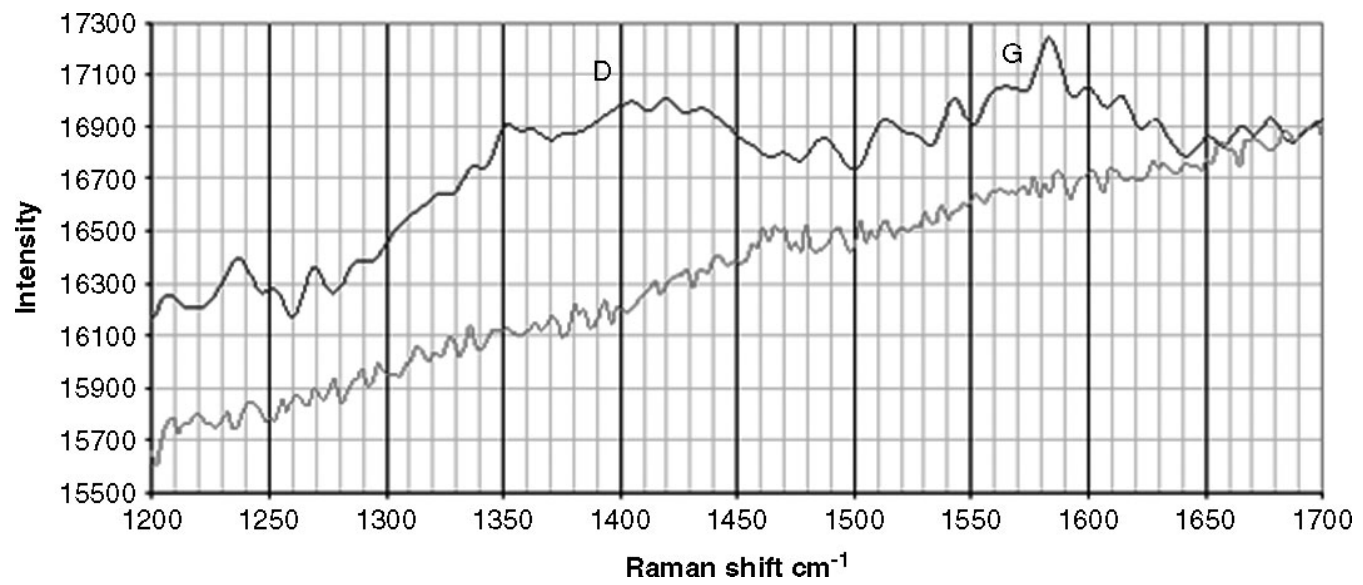

Fig 9. Micro-Raman spectra of the fullerene-coated scanning probe tip (dipped in $\mathrm{PdCl}_{2}$ ), before (red) and after (blue) the synthesis experiment.

(Robertson, 2007). The peaks for $\mathrm{G}$ and $\mathrm{D}$ band are of comparable intensity, which is indicative of MWCNT being synthesized in this process. However, it can be argued that since the $\mathrm{G}$ band has a higher intensity than D band-purely SWCNT are synthesized. The Raman data is also indicative of semiconducting (or mixture of conducting and semiconducting) SWCNT being synthesized in this process.

\section{Conclusion}

This investigation provides the proof of concept for the synthesis of SWCNT and possibly MWCNT on scanning probe tips using dip pen techniques. This technique obviates CVD apparatus while enabling synthesis of CNT under ambient conditions. By coating the tips with fullerene and metal catalyst precursors, the process enables the synthesis of CNT without requiring any process gas flow (except for maintaining an inert atmosphere) while the synthesis temperatures can be as low as $350^{\circ} \mathrm{C}$. The SEM images show that the synthesized CNT are constrained to a localized area near the scanning probe tip. The process presented in this study proves the feasibility for developing a portable CNT synthesis platform (or instrument) where CNT synthesis can be performed "on demand".

The Raman spectroscopy showed peaks in the RBM mode in the range of $100-300 \mathrm{~cm}^{-1}$, and the $\mathrm{D}$ and $\mathrm{G}$ peaks were observed at 1350 and $1582 \mathrm{~cm}^{-1}$ respectively. The RBM data shows the SWCNT diameter ranges from 0.9 to $1.5 \mathrm{~nm}$. The intensity of $\mathrm{D}$ band was slightly higher than the $G$ bands, suggesting the existence of MWCNT, while the presence of the RBM mode is indicative of SWCNT. Hence this process can be optimized to synthesize either SWCNT or MWCNT 
on the scanning probe tips. The process can be optimized for growing CNT of a single chirality (i.e. metallic or nonmetallic SWCNT).

\section{Acknowledgments}

The authors gratefully acknowledge the guidance and financial support by Dr. Amit Lal from the Micro Technology Office (MTO) of the Defense Advanced Research Projects Agency (DARPA). The authors also gratefully acknowledge the financial support and monitoring by Dr. Ryan Lu, Mr. Richard Nguyen and Mr. Al Navasca from the Space and Naval Warfare Center (SPAWAR). Support from the Office of Naval Research (ONR) is also acknowledged. Support from the Mechanical Engineering Department at Texas A\&M University and the Texas Engineering Experimentation Station (TEES) is also gratefully acknowledged. The FE-SEM acquisition was supported by the National Science Foundation under Grant No. DBI-0116835.

During the course of execution of this study, the authors and their research activities were also supported partially through various other research programs and are duly acknowledged here: National Science Foundation (CBET Grant No. 0630703), Micro/Nano-Fluidics Fundamental Focus Center (DARPA-MF3) through the University of California at Irvine, New Investigations Program (NIP 2005) of the Texas Space Grants Consortium (TSGC), Office of Naval Research (ONR), Air Force Office of Scientific Research (AFOSR) through the American Society for Engineering Education (ASEE), the Summer Faculty Fellowship (SFFP) Program at the Air Force Research Labs. (AFRL), Army Research Office (ARO) SBIR Phase II subcontract through Lyntech Inc. and the National Science Foundation (NSF) SBIR Phase I through NanoMEMS Research LLC. We also thank Dr. S. Sinha from the Department of Physics at the University of New Haven for helpful discussions on catalysis and CVD of CNT. The help rendered by Mr. Manoj Sunder, graduate student of Mechanical Engineering at Texas A\&M University in preparing Figure 3 is also acknowledged.

\section{References}

Banerjee D, Amro N, Disawal S, Fragala J: Optimization of microfluidic ink-delivery apparatus for Dip Pen Nanolithography. J Microlith, Microfabric Microsystems 4, 23014-23021 (2005).

Chiang LY, Swirczewski JW, Hsu CS, Chowdhury SK, Cameron S, Creegan K: Multi-hydroxy additions onto CG0 fullerene molecules. J Chem Soc Chem Commun 24, 1791-1793 (1992).

Cox D, Behal S, Disko M, Gorun S, Greaney M, et al.: Characterization of C60 and C70 clusters. J Am Chem Soc 113, 2940-2944 (1991).

Ebbesen T, Ajayan P: Large-Scale synthesis of carbon nanotubes. Nature 358, 220-222 (1992).

Hafner J, Cheung C, Lieber C: Growth of nanotubes for probe microscopy tips. Nature 398, 761-765 (1999).

Iijima S: Helical microtubules of graphitic carbon. Nature 354, 56-58 (1991).

Jorio A, Saito R, Dresselhaus G, Dresselhaus M: Determination of nanotubes properties by Raman spectroscopy. Philos Trans $R$ Soc Lond Part A 362, 2311-2336 (2004).

Jorio A, Saito R, Hafner J, Lieber C, Hunter $\mathrm{M}$, et al.: Determination of isolated single-wall carbon Nanotubes by resonant Raman scattering. Phys Rev Lett 86, 1118 (2001).

Li Y, Kim W, Zhang Y, Rolandi M, Wang D, et al.: Growth of single-walled carbon Nanotubes from discrete catalytic Nanoparticles of various sizes. J Phys Chem B 105, 11424-11428 (2001).

Mochida I, Egashira M, Koura H, Dakeshita K, Yoon S, et al.: Carbonization of C60 and C70 fullerenes to fullerene soot. Carbon 33, 1186-1188 (1995).

Ouellette J: Building the Nanofuture with Carbon Tubes. The Industiral Physicist, December 18-21 (2002).

Robertson J: Growth of nanotubes for electronics. Materials Today 10(1-2), 36-45 (2007).

Rosner B, Duenas T, Banerjee D, Shile R, Amro N: Rendlen: functional extensions of Dip Pen Nanolithography: active probes and microfluidic ink delivery. J Smart Mater Struct 15, S124-S130 (2006).

Singer M, Grumer J: Carbon formation in very rich hydrocarbonair flames. Proc Combust Inst 7, 559 (1959).

Su DS, Chen XW: Natural lavas as catalysts for efficient production of carbon nanotubes and nanofibers. Angew Chem Int Ed Engl 46, 1823-1824 (2007).

Sunden E, Wright T, Lee J, King W, Graham S: Roomtemperature chemical vapor deposition and mass detection on a heated atomic force microscope cantilever. Appl Phys Lett 88, 33107-33110 (2006).

Thomsen C, Reich S, Maultzsch J: Resonant Raman spectroscopy of nanotubes. Philos Trans $R$ Soc Lond Part A 362, 2337-2359 (2004). 